\title{
Sliding-MOMP Based Channel Estimation Scheme for ISDB-T Systems
}

\author{
Ziji Ma, ${ }^{1}$ Shuaifeng Guo, ${ }^{1}$ Hongli Liu, ${ }^{1}$ and Minoru Okada ${ }^{2}$ \\ ${ }^{1}$ College of Electrical and Information Engineering, Hunan University, Changsha, Hunan 410082, China \\ ${ }^{2}$ Graduate School of Information Science, Nara Institute of Science and Technology, Ikoma, Nara 630-0101, Japan \\ Correspondence should be addressed to Ziji Ma; zijima@hnu.edu.cn
}

Received 5 February 2016; Revised 25 May 2016; Accepted 31 May 2016

Academic Editor: Xiumei Li

Copyright (c) 2016 Ziji Ma et al. This is an open access article distributed under the Creative Commons Attribution License, which permits unrestricted use, distribution, and reproduction in any medium, provided the original work is properly cited.

\begin{abstract}
Compressive sensing based channel estimation has shown its advantage of accurate reconstruction for sparse signal with less pilots for OFDM systems. However, high computational cost requirement of CS method, due to linear programming, significantly restricts its implementation in practical applications. In this paper, we propose a reduced complexity channel estimation scheme of modified orthogonal matching pursuit with sliding windows for ISDB-T (Integrated Services Digital Broadcasting for Terrestrial) system. The proposed scheme can reduce the computational cost by limiting the searching region as well as making effective use of the last estimation result. In addition, adaptive tracking strategy with sliding sampling window can improve the robustness of CS based methods to guarantee its accuracy of channel matrix reconstruction, even for fast time-variant channels. The computer simulation demonstrates its impact on improving bit error rate and computational complexity for ISDB-T system.
\end{abstract}

\section{Introduction}

Orthogonal frequency division multiplexing (OFDM) that is a multicarrier modulation technique has been adopted by ISDB-T (Integrated Services Digital Broadcasting for Terrestrial) system [1] due to its robustness against multipath interference and high spectrum availability rate. In ISDB-T systems, a dynamic and accurate channel estimation method is normally essential for demodulation, synchronization, equalization, and so on. Generally, pilot-aided channel estimation schemes are employed to recover the channel impulse response in ISDB-T systems. Recently, a new technique named compressive sensing (CS), which can reliably recover the original sparse signal from very limited samples, has been exploited for sparse channel estimation. With the definition of the restricted isometry property (RIP) being introduced, the concept of CS was firstly proposed in 2006 [2, 3]. After that, the CS has been intensely investigated for its better reconstruction performance and wide application field [47]. For channel estimation, CS based approaches can obtain more accurate estimation with the same number of pilots or similar estimation performance with less pilots in contrast to least square (LS) based approaches [8].
For CS based channel estimation methods, an important assumption of sparse multipath channel, where a multipath propagation channel can be separated into a few strong paths and many uncountable weak paths whose transmission energy is approximate to zero, should stand in practical application. In other words, most taps of a channel are negligible, as estimating channel impulse response (CIR). For ISDB-T system, this assumption is commonly acceptable as an engineering approximation $[9,10]$, and it is also assumed to be true in this paper. Thus, the discussion about the sparsity of propagation channels is beyond the research range of the paper.

Bajwa et al. in [10] have proposed several basic CS based channel estimation methods by using DS solution for varying communication models, including single-antenna and multiantenna systems. Berger has detailedly introduced the CS based sparse channel estimation, including technical background, basic definitions, algorithm, and practical application [8]. In [11], CS based channel estimation is employed for underwater acoustic communication and compares its bit error ratio performance with other methods. Based on conventional matching pursuit algorithm [12], Troop and Gilbert have proposed an orthogonal matching pursuit 
(OMP) algorithm to solve the linear program for CS, which can significantly reduce the computational complexity to recover original sparse signal in contrast to basic pursuit based methods [13]. In order to further decrease the requirement of computational cost, an MOMP algorithm has been proposed to further decrease the computational complexity of OMP by limiting redundant correlation calculation [14, 15]. Meng et al. proposed a low-cost and high-resolution channel estimator by using special pilot design [16]. Undoubtedly, these methods that rearrange the pilot subcarriers are not available for the ISDB-T system of current version [1]. More recently, Paderna et al. has proposed an MOMP based channel estimation method to overcome imperfect synchronization via processing received signal in time-domain instead of frequency-domain $[17,18]$. However, in consideration of the requirement of low computational complexity, for high-speed moving reception, accurately obtaining channel's FIR by using CS based methods is still a big challenge due to severe interference from Doppler frequency shift and multipath propagation.

In this paper, we further improve the conventional MOMP algorithm by setting a flexible searching range to enhance its robustness. An improved channel estimation scheme based on sliding-MOMP (SMOMP) has been proposed, which can adaptively deal with fast and flat fading channel with relatively low calculation complexity. Also, we introduce a reconstruction method of channel matrix with the derivation of initial phases. The remaining part of this paper is organized as follows. Section 2 introduces the basis system model, and Section 3 gives a simple review of the CS based channel estimation methods. The proposed SMOMP scheme is introduced in detail in Section 4. The numerical results and conclusions are presented in Sections 5 and 6, respectively.

\section{System Model}

Consider an uncoded OFDM system with $N$ subcarriers and $N_{\mathrm{CP}}$ cyclic prefix (CP) as guard interval (GI). The constellated data stream $S$, including transmission data $S_{d}$ and pilot subcarriers $S_{p}$, has been transformed by $N$-point inverse discrete Fourier transform (IDFT) from serial to parallel signal. After inserting a CP, the transmission signal $x(n)$ can be written as

$$
x(n)=\frac{1}{N} \sum_{k=0}^{N-1} S(k) e^{j 2 \pi n k / N}, \quad-N_{\mathrm{CP}} \leq n<N .
$$

Assume that the synchronization is perfect and the CP is longer than the channel's maximum delay. Then, the received signal $y(n)$, after the removal of CP, can be presented as

$$
y(n)=\sum_{l=0}^{L-1} h(n ; l) x(n-l)+w(n)=H_{t} F^{H} S+w,
$$

where $H_{t}$ denotes a time-domain channel matrix, $F^{H}$ denotes a complex conjugate transpose of an $N \times N$ unitary DFT matrix, and $w$ denotes the additive white Gaussian noise (AWGN). For high-speed moving reception of
ISDB-T systems, the transmission signal is interfered by a time/frequency doubly selective multipath fading channel whose characteristic parameters are time-variant. Without loss of generality, we here exploit the basis expansion model (BEM), which commonly describes a random channel with less second-order diversity than numerous channel parameters over a basis of complex exponentials, to model a timevariant channel [19]. The BEM can clearly show a channel dictionary of sparse pattern (SP) of the angle-delay-Doppler spread with independent diversities on angle, time, and frequency order. A propagation channel tap $h(n ; l)$ is given by BEM with time-variant impulse response, $l \in[0, L)$ where $l$ presents the $l$ th transmission tap among at most $L$ channel paths:

$$
h(n ; l)=\sum_{q=-Q}^{Q} c_{q}(l) e^{j \phi} e^{j 2 \pi n q / N},
$$

where $Q$ is the $q$ th BEM amplitude coefficient of the $l$ th channel tap, $c_{q}(l)$ is the BEM model order, and $\phi$ that denotes the initial phase of the tap following a uniform distribution in $[0,2 \pi]$. The maximum Doppler spread is given by $Q /\left(T_{c} N\right)$. After removing GI, the received signal that is transformed to frequency-domain by using DFT is presented by

$$
R(k)=F H_{t} F^{H} S+F w=H_{f} S+v,
$$

where $H_{f}$ denotes the frequency-domain channel matrix. The pilot subcarriers $R_{p}$ are extracted from the $R(k)$ to derive the FIR at pilot positions $H_{p}=R_{p} / S_{p}$. Then, we can use a kind of filter or interpolator to reconstruct a channel matrix, which is normally called least squares (LS) based estimation method. However, due to the effect of timeDoppler spread, the so-called intercarrier interference (ICI) destroys the subcarriers' othogonality of the OFDM signal, which causes the performance declination of ISDB-T systems.

\section{CS Based Channel Estimation}

As mentioned before, in contrast to other channel estimation methods, especially LS based methods, CS based approaches can recover the CIR via much less pilot subcarriers for OFDM systems. As a result, in $[10,20]$, if the actual or effective number of DoF (Degree of Freedom) is much less than the maximum number of DoF in a channel, a channel can be defined as a "sparse" one. For sparse channel estimation, the recovery results by CS based methods are the channel characteristic parameters, that is, channel taps, Doppler frequency offset, channel delay, and distorted phase. Then, the channel matrix can be reconstructed depending on the recovered results. In $[2,3]$, the authors give the definition of CS and two important preconditions, RIP and uncorrelated measurement vectors. Moreover, Berger et al. summarizes the CS based channel estimation methods [8]. Consider a standard linear measurement model:

$$
r=\Psi \theta+\epsilon,
$$

where $r$ is an $M$-vector of responses that can be represented in an arbitrary basis, $\psi_{k k=1}^{n}$, with the weighting coefficients 
$\theta$. $\epsilon$ is an $M$-vector of random noise with variance $\delta^{2}$. In general, $\theta$ is an unknown $P$-vector, and $\Psi=\left(\psi_{1}, \psi_{2}, \ldots, \psi_{p}\right)$ denotes an $M \times P(M<P)$ measurement matrix. When CS based channel estimation is used for OFDM systems, the measurement matrix commonly consists of some columns of a 2-dimensional DFT matrix that obeys the RIP condition due to the orthogonality of rows and columns. According to the RIP, the isometry constant $\delta_{S}$ of $\Psi$ as the smallest number such that

$$
(1-\delta)\|\theta\|_{2}^{2} \leq\|\Psi \theta\|_{2}^{2} \leq(1+\delta)\|\theta\|_{2}^{2}
$$

holds for all $X$-sparse vectors, another representation of $X$ actual DoFs, where $\|\cdot\|_{2}$ is denoted as the $\ell_{2}$-norm. For frequency-selective fading channel, using 1-dimensional DFT matrix is usually effective and reliable for signal reconstruction. However, in the case of doubly selective fading channel, a 2-dimensional DFT matrix is essential. At the receiver, one can derive $h$ to obtain an estimate. Here, we have to highlight an easily overlooked point that the solution result of CS is not an estimate of a channel matrix, but a CIR matrix, for 2D case, which represents the path's characters (delay-angle-Doppler shift) of a multipath channel. In general, the CS problem can be solved by two kinds of approaches, basis pursuit (BP) and matching pursuit (MP) [21]. The former is an $\ell_{1}$ norm based approach that has some well-known algorithms, for example, LASSO and DS (Danztig Selector) [22-24]. The latter is a dynamic programming based greedy algorithm. However, no matter which approach we take, the computational cost is quite high due to large-scale matrix calculations.

3.1. Orthogonal Matching Pursuit. The conventional OMP is a greedy algorithm that can solve the CS with relatively low computational complexity, in contrast to most BP based approaches and original MP. The details of OMP are introduced in $[12,13]$. OMP aims to solve a sparse LP problem without considering any impact of noise for which (5) would be rewritten as, $r=\Psi \theta$, where $r, \Psi$, and $\theta$ have the similar definitions introduced before. As an iterative algorithm, OMP aims to detect the columns of $\Psi$ in a greedy fashion, which is most strongly correlated with the remaining part of $r$. Then, the column with maximum correlated value would be extracted from $\Psi$, while the residual part would be reserved to the next iteration. After $P$ iterations, the algorithm will have identified the correct set of columns. Also, one can get an identified matrix and a location index set, both of which would be used to reconstruct a new estimate.

\section{Proposed Scheme}

The procedure of CS based channel estimation consists of two steps, (1) solving the CS problem and (2) reconstructing the channel matrix. It is worthy of noticing that research about the latter is seldom involved in most literatures. Actually, the results of CS problem are normally not equivalent to the channel estimates. Taking account of the trade-off between reducing the computational complexity and increasing the reconstruction performance, the proposed scheme improves the conventional OMP based channel estimation schemes with the following approaches.

4.1. Measurement Matrix. The Fourier matrix is a natural measurement matrix for the incoherency and orthogonality characteristic for the OFDM system of ISDB-T. Because of aiming to deal with the time/frequency doubly selective timevariant channel, the measurement matrix is comprised of 2dimensional (frequency and time axis) DFT matrix to detect delay-Doppler spread. Consider a $N_{t} N_{f} \times K D$ measurement matrix $\Psi$, as an example, is given below:

$$
\begin{gathered}
\Psi=\left[\begin{array}{llll}
w_{N_{t}}^{n K / 2} & w_{N_{t}}^{n(K / 2-1)} & \cdots & w_{N_{t}}^{-n K / 2}
\end{array}\right] \\
\otimes\left[\begin{array}{llll}
1 & w_{N_{f}}^{n 1} & \cdots & w_{N_{f}}^{n(D-1)}
\end{array}\right],
\end{gathered}
$$

where $\otimes$ is denoted as the Kronecker product and $w_{N}^{k}=$ $e^{-j 2 \pi k / N}$, that is, the entry of DFT matrix. $N_{t}$ and $N_{f}$ denote the time and frequency separation, which are usually equal to the number of pilots in time- and frequency-domain. Meanwhile, $K$ and $D$ denote the maximum resolvable division of Doppler shifts and channel delays, respectively. In order to guarantee the detecting accuracy, $\Psi$ is a big size matrix that requires large-scale matrix calculations. Therefore, reducing the size of $\Psi$ can directly improve the computational cost. In general, $D$ is defined as the length of a whole OFDM symbol to detect long enough channel delay. Consider that the assumption of long enough CP is true in most cases. Thus, the length of $D$ can be shortened. In our case, $D$ is set to the length of CP and it works well. With the same reason, if the maximum Doppler shift is known previously, $K$ also can be shortened to $K_{\min }$ to decrease the size of $\Psi$ further. Thus, (7) can be rewritten as

$$
\begin{aligned}
\Psi= & {\left[\begin{array}{llll}
w_{N_{t}}^{n K_{\min } / 2} & w_{N_{t}}^{n\left(K_{\min } / 2-1\right)} & \cdots & w_{N_{t}}^{-n K_{\min } / 2}
\end{array}\right] } \\
& \otimes\left[\begin{array}{llll}
1 & w_{N_{f}}^{n 1} & \cdots & w_{N_{f}}^{n\left(N_{\mathrm{CP}}-1\right)}
\end{array}\right] .
\end{aligned}
$$

Then, the size of measurement matrix is decreased to $N_{t} N_{f} \times$ $K_{\text {min }} N_{\mathrm{CP}}$. In most cases, the decreased measurement matrix is suitable and effective for the proposed scheme. In addition, increasing the flexibility of the size of the measurement matrix in terms of the maximum channel delay to realize an adaptive scheme is also an attractive alternative.

4.2. Modified Matching Pursuit. For a time/frequency double selective fast-fading channel, the channel characteristics coefficients, including maximum Doppler frequency and the number of propagation paths, usually hardly change during a short temporal interval. In this paper, some extreme cases, for example, getting in or out of a tunnel, are beyond our discussion. For the ISDB-T system, the characteristics coefficients of the current OFDM symbol seldom change too much between adjacent several OFDM symbols. Yet the conventional OMP needs to calculate correlation value of each entry's pair between $\Psi$ and $r$, where the correlation calculation mostly dominates the complexity cost of the OMP [8]. It is worthy of mentioning that, in a sparse system, it is reasonable to 
neglect the effect from weak paths that are represented as corresponding columns of measurement matrix $\Psi$. Therefore, we just need to calculate the correlation values of nonzero elements and their adjacent elements that are indexed in the location index set of the last estimate. So do the measurement matrix's columns. The index set, which can be reused to decrease the calculation time upon correlation operation, is upgraded after an OMP iterative loop. In other words, this procedure is equivalent to decreasing the times of correlation calculations, which is definitely helpful to reduce the overall computational cost of signal recovery. Additionally, notice that the sparsity level is an essential input parameter for the conventional OMP. However, the number of the propagation paths is an unknown variable before getting an estimate, and it is also a changeable parameter for time-variant channels. Our proposed MOMP sets an adaptive parameter of iterative loop to improve the algorithm's flexibility and robustness. The flowchart of the proposed MOMP is shown in Figure 1.

The procedure is as follows:

(1) As the initial setting, a $M \times P$ measurement matrix $\Psi$ is given in terms of the target system, and the detection loop counter $i$ that is marked as superscript is set to 0 . Initial index set $\operatorname{In}^{0}=[1,1, \ldots, 1]_{1 \times P}$.

(2) Initialize the residual vector $r_{1}^{i}=r^{i}$, the index set $\operatorname{In}_{1}^{i}=\emptyset(i \neq 0)$, the sensing matrix $\Phi_{1}=$ $\emptyset$, and the iteration counter $t=1, t \in[1, M]$. Meanwhile, a threshold parameter is defined as $\mathrm{Th}^{i}=$ $(\alpha / M) \sum_{k=0}^{M-1}\left|r^{i}(k)\right|^{2}$, where $\alpha \in(0,1]$ denotes a proportional coefficient.

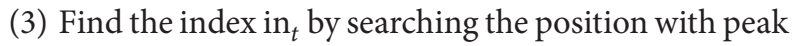
value over power threshold via correlation calculation, where the detection range is the last index set $\operatorname{In}^{i-1}$ :

$$
\operatorname{in}_{t}=\arg \max _{k \in \operatorname{In}^{i-1}}\left|\left\langle r_{t-1}^{i}, \psi_{k}\right\rangle\right|
$$

(4) Make a new index set $\operatorname{In}_{t}^{i}=\operatorname{In}_{t-1}^{i} \cup$ in ${ }_{t}$ and extract the corresponding columns from measurement matrix $\Psi_{t-1}^{i}$ to insert them into the last one, and augment it to a new sensing matrix as follows:

$$
\Phi_{t}^{i}=\left[\Phi_{t-1}^{i} \psi_{\mathrm{in}_{t}}\right]
$$

(5) Solve a LS problem to obtain a new signal estimate:

$$
\widehat{\theta}_{t}=\arg \min \left\|r^{i}-\Phi \theta\right\|_{2} .
$$

(6) Calculate the new approximation of the data and the new residual sequence:

$$
r_{t}^{i}=r^{i}-\Phi_{t} \hat{\theta}_{t}
$$

(7) If the residual power is lower than the threshold $P_{r_{t}^{i}}<$ $\mathrm{Th}^{i}$ or the iterative counter is beyond the maximum iterative times $t \geq M$, the iteration procedure will end. Otherwise, return to Step (2) and start a new iterative step.
(8) The estimate result $\widehat{\theta}^{i}=\widehat{\theta}_{t}$ and an upgraded index set $\mathrm{In}^{i}$ are saved as output signal and sent forward to the next detection loop as well.

Remark the following:

(1) Only for the initial detection is the index set set to one vector with all ones, $\operatorname{In}^{0}=[1,1, \ldots, 1]_{1 \times P}$, for which the correlation calculation traverses the overall $\Psi$. From the second detection loop, an upgraded $\mathrm{In}^{i}$ that consists of a lot of zero and a few one elements can be reset according to the last index set. If the element at $\operatorname{In}^{i}(k)$ position is zero, the correlation calculation and other related processes will be neglected.

(2) In order to detect the quickly changing channel characteristic coefficients in the next loop, it is necessary to guarantee a modest flexibility of the index set $\operatorname{In}_{t}^{i}$ to track the CIR, as well as reducing the nonzero elements of it. The diagram of the upgraded process of the index set $\operatorname{In}^{i}$ is shown in Figure 2. $\operatorname{In}^{i}$ is renewed as

$$
\operatorname{In}^{i}(k)= \begin{cases}1 & g(k) \neq 0 \\ 0 & g(k)=0\end{cases}
$$

(3) Except for some special cases, the difference of channel characteristic coefficients between two adjacent OFDM symbols is usually very limited and has little sudden change. In other words, detecting a merely small range, front and back positions, around the last estimated CIR location can effectively track the change of a fast fading channel. The faster the CIR changes, the larger the extending unit $V$ that is set to 1 in Figure 2 from $\widehat{\theta}^{i}$ to $g$. Also, in consideration of the robustness of the algorithm, an extending parameter $V$ is dominated in terms of the power level of propagation paths. After that, we use a scattered map of $g$ to derive a new index set $\operatorname{In}^{i}$. The extending unit $V$ is the Euclidean distance around every nonzero $\hat{\theta}^{i}$. As $V=1$, the processing of getting the map of $g$ is obtained by

$$
\begin{aligned}
g(k)= & \frac{1}{2} \widehat{\theta}^{i}(k-N-1)+\frac{1}{2} \widehat{\theta}^{i}(k-1)+\widehat{\theta}^{i}(k) \\
& +\frac{1}{2} \hat{\theta}^{i}(k+1)+\frac{1}{2} \widehat{\theta}^{i}(k+N+1) .
\end{aligned}
$$

(4) The residual power $P_{r_{t}^{i}}$, the average power of residual vector $r_{t}^{i}$, is defined as $P_{r_{t}^{i}}=(1 / M) \sum_{k=0}^{M-1}\left|r_{t}^{i}(k)\right|^{2}$. If most of high-power propagation paths have already been detected and extracted from $r^{i}, P_{r_{t}^{i}}$ will be a small value. Thus, a detection threshold $\mathrm{Th}^{i}$ that is adjusted by the proportional coefficient $\alpha$ is set to control the detection process. As the residual power $P_{r_{t}^{i}}$ is lower than the threshold $\mathrm{Th}^{i}$, an iteration loop does end. 


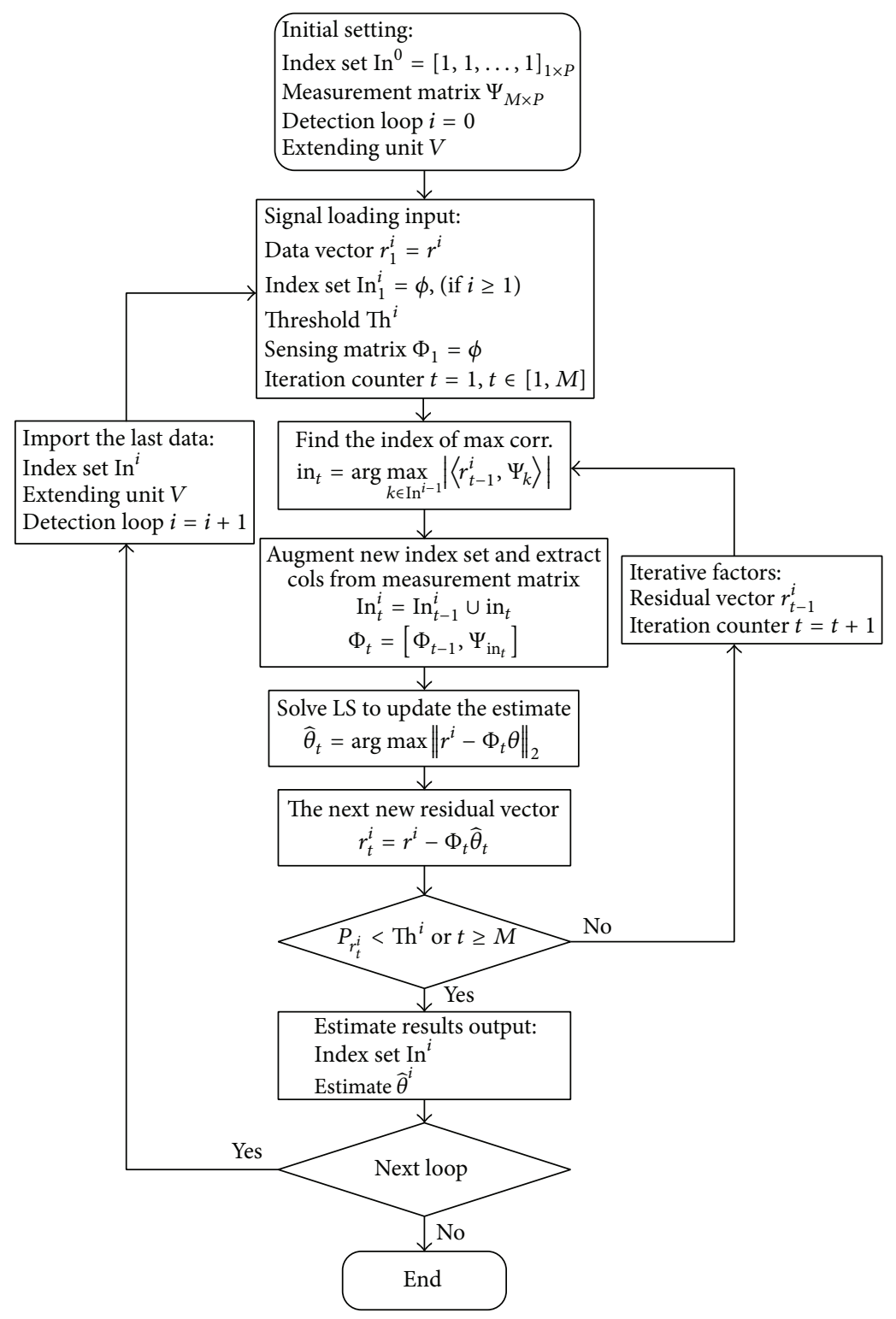

FIGURE 1: Flowchart of modified orthogonal matching pursuit.

(5) In consideration of the effect of suddenly changing channels, such as entering and driving out of a tunnel, MOMP has to periodically search the full measurement matrix $\Psi$ by resetting the index set $\operatorname{In}^{i}$ as one vector to track the channel's sudden change. Thus, an adaptive parameter $\rho, \rho \in(0,1]$, is introduced to adjust the ratio of repeating search.

In brief, the MOMP can effectively reduce the redundant correlation calculation of OMP and robustly deal with both fast and flat fading channel. Working with the optimized measurement matrix together, the MOMP can run much faster than the conventional OMP and nearly not take any accuracy loss of estimate.

4.3. Reconstruction of Channel Matrix. The reconstruction methods of original signal based on CS have been introduced in many literatures, but another important issue, the reconstruction of channel matrix, is seldom discussed. According to the general understanding $[2,8,10]$, the reconstruction of CS is equivalent to finding a suitable dictionary for sparse representation of channels, which definitely is a very important research objective. However, an ISDB-T reception system, in most cases, requires an accurate estimate of channel matrix rather than a reconstructed dictionary for representing the channel's sparsity. Therefore, an improvement on reconstructing channel matrix can further increase the accuracy of channel estimation, and provide effective support to improve the ISDB-T system's performance. Since the calculation result of CS algorithm with 2-dimensional measurement matrix is a sparse complex matrix, then from the sparse matrix, the very limited "big value" elements, whose module, argument, and position are regarded as FIR information, are extracted to reconstruct channel matrices. 

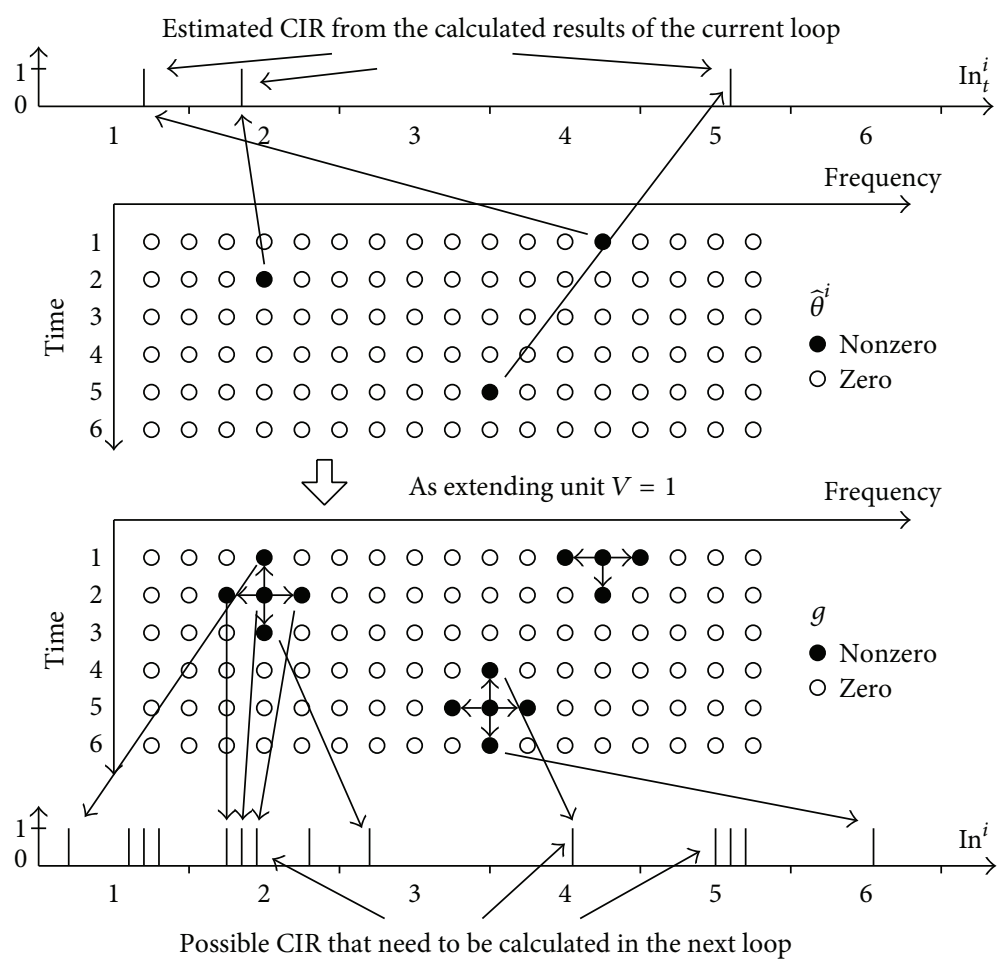

FIGURE 2: Reconstruction of index set with extending parameter $V=1$.

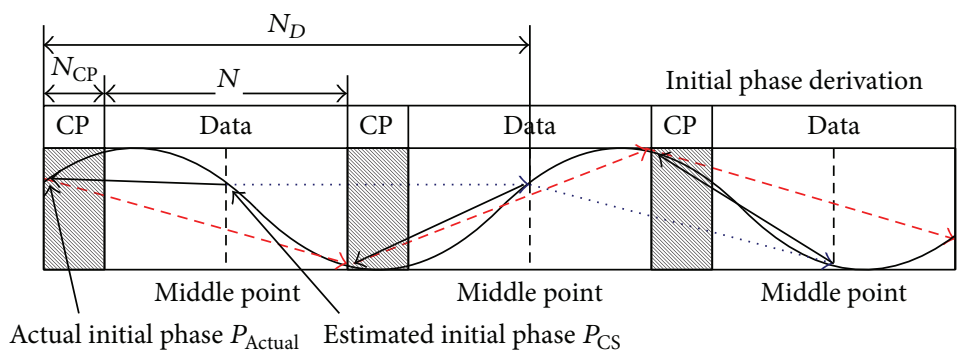

FIGURE 3: The derivation of the initial phase of a propagation path in terms of the CS based estimates.

The energy level of propagation paths is almost proportional to the module of those elements, while the channel delay and Doppler shift level can be derived from the position of those elements subject to a particular reconstruction dictionary. However, it is not accurate enough to get the initial phase of propagation paths by directly using the element's argument of the sparse matrix. As a linear programming method, CS gives a statistical optimum solution that obeys the piecewise linear assumption $[25,26]$. The element's argument generally represents a phase $P_{\mathrm{CS}}$ at the middle point of an OFDM symbol, which is shown in Figure 3. Therefore, the actual initial phase $P_{\text {Actual }}$ of the current propagation path, in the case of $N_{\mathrm{CP}}=N / 4$, can be approximately derived by

$$
\begin{aligned}
P_{\text {Actual }} & =P_{\mathrm{CS}}-\frac{2 \pi}{N_{D}}\left(N_{\mathrm{CP}}+N\right) \frac{N_{\mathrm{CP}}+N / 2}{N_{\mathrm{CP}}+N} \\
& =P_{\mathrm{CS}}-\frac{\pi\left(N_{\mathrm{CP}}+N\right)}{N_{D}},
\end{aligned}
$$

where $N$ and $N_{D}$ are the length of one OFDM symbol and the discrete length of Doppler shift, respectively. After that, the modified initial phase $P_{\text {Actual }}$ and other estimated CIR coefficients are substituted into (3) to reconstruct the channel matrix. Instead of $P_{\mathrm{CS}}$, using $P_{\text {Actual }}$ can effectively increase the accuracy of channel matrix reconstruction and improve the BER performance of ISDB-T system.

4.4. Sliding-MOMP. The MOMP focuses on reducing the computation complexity, while in this section we propose an iterative method with adaptive sliding window to improve the accuracy of MOMP further, especially for time-variant fast fading channels. In order to track the quickly changing channel coefficients due to high-speed mobility reception, the size of the measurement matrix that is derived from a $2 \mathrm{D}$ DFT matrix has to been increased with the delay-Doppler spread. With respect to the size of $\Psi$, the MOMP executes a block-estimation after receiving $N_{t}$ OFDM symbols, but not for each OFDM symbol. However, for variable speed 


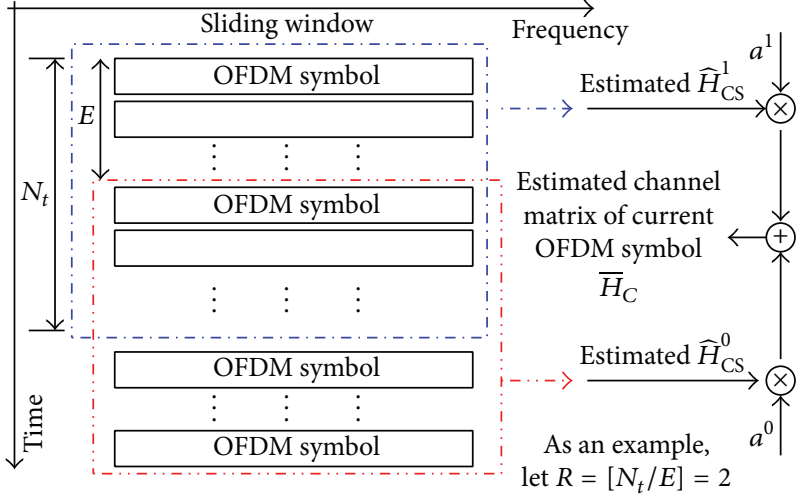

FIGURE 4: The sliding-MOMP method with sliding overlapped window.

mobility reception, only increasing the size of $\Psi$ is quite inadequate to track the channel's change. Since CS is a linear programming based algorithm, the estimate's accuracy of MOMP is significantly degraded compared to steady highspeed mobility reception.

In addition, another problem about spread of estimate error needs to be faced. The channel matrix is reconstructed on the basis of the estimated FIR results. However, the speed of estimator is slower than that of channel coefficients, if the block-estimation method is employed. The estimate error due to the variable speed mobility reception spreads into the adjacent channel matrices, even if the error is slight.

We use a sliding window to adjust the execution frequency of MOMP, where the window's width and sliding step length are, respectively, defined as $N_{t}$ and $S_{L}, S_{L} \leq N_{t}$. $S_{L}$ is set to a suitable value to match the changes of delayDoppler spread. As $S_{L}=N_{t}$, the proposed iterative method does not work. In the case of $S_{L}<N_{t}$, the sliding windows are overlapped. One OFDM symbol thus executes $R$ times of MOMP, where the iterative depth $R$ is derived by $R=$ $\left\lceil N_{t} / S_{L}\right\rceil$ (operator $\lceil\cdot\rceil$ denotes a roundup operation). The current channel matrix then is given by

$$
\bar{H}_{c}=\sum_{k=0}^{R} a^{(k)} \widehat{H}_{\mathrm{cs}}^{(k)},
$$

$$
\text { subject to } \sum_{k=0}^{R} a^{(k)}=1 \text {, }
$$

where $\bar{H}_{c}, \widehat{H}_{\mathrm{cs}}^{(k)}$, and $a^{(k)}$ are denoted as the current channel matrix, the reconstructed channel matrix from $k$ th MOMP, and the normalized weight of that estimate, respectively. We set $a_{i} / \sum_{k=i+1}^{R-1} a_{k}=g$, where $g$ is a proportionality constant. The weight of the latest estimate increases with increasing $g$. The process of the iterative method with sliding windows is shown in Figure 4, in which we set $R=2$, and the estimate of the current MOMP and the last MOMP is denoted as $\widehat{H}_{\mathrm{cs}}^{0}$ and $\widehat{H}_{\mathrm{cs}}^{1}$, respectively.

As the ISDB-T receiver is on a variable speed moving status, we have to increase the MOMP's execution frequency
TABLE 1: Simulation parameters.

\begin{tabular}{lc}
\hline FFT size $(N)$ & $64,128,256,512,1024$, and 2048 \\
Guard interval & $N / 4$ \\
Pilot type & Scattered type \\
Modulation type & QPSK \\
Normalized Doppler $\left(F_{d} T_{s}\right)$ & $0.03125,0.0625$, and 0.125 \\
Noise type & AWGN \\
\hline
\end{tabular}

$R$ to update the quickly changing estimates. Conversely, we decrease the value of $R$ for steady mobility reception, until $R=1$ for uniform-speed reception.

\section{Numerical Results}

In this section, we present simulation results to demonstrate our proposed scheme's performance. We employ an uncoded OFDM system with QPSK constellation, in which the main parameters are shown in Table 1, similar to the mode 1 of ISDB-T but without encoding and interleaving procedure. A random pilot assignment or other especial pilot design approaches are better options to gain the optimum performance according to the CS theory. But ISDB-T standard has its specific pilot arrangement (see [1]), for which the performance of the proposed scheme is slightly worse than the optimal performance of CS. We choose the Typical Urban 6 paths (TU6) model (in [27, Annex C3.1]) as a double selective fading channel model. In our numerical simulation, the TU6 delay parameters, power and delay, have been normalized with the total signal. However, this model cannot perfectly simulate a channel of variable speed mobility reception. Thus, 2 key channel parameters, delay time and Doppler spread, are set to variables ones with a given proportion value. In order to obtain relatively fair comparative conclusions, we adopt 2 simple equalizers, Zero-Forcing (ZF) and banded minimum mean squared error (MMSE), for varying schemes.

We firstly compare the computational complexity of the proposed MOMP with the conventional OMP and DS method that is solved by the simplex algorithm [28]. With the increase of FFT size, the execution time of all 3 methods quickly increases. In contrast to the DS method, both OMP and MOMP have clear advantage on computational complexity, which is shown in Figure 3. Besides simplex algorithm, interior point algorithm [29] has also been exploited to solve the BP problem, but it cannot clearly improve the computational complexity either. As mentioned before, the computational complexity of OMP is mainly dominated by the correlation calculation. Assume that $\Psi$ is a $N \times M$-matrix with $S$ nonzero vectors and repeating ratio parameter $\rho$. Then, it only costs $(1-\rho) N S^{2}+\rho N M^{2}$ complex multiplications for proposed MOMP instead of $N M^{2}$ complex multiplications for OMP. Since $S \ll M$ and low $\rho$, the MOMP can significantly improve the performance on computational complexity with decrease of a channel's DoF. According to the introduction to the proposed SMOMP, its computational complexity increases with the iterative depth $R$. In consideration of the definition of $R$, the computational complexity 


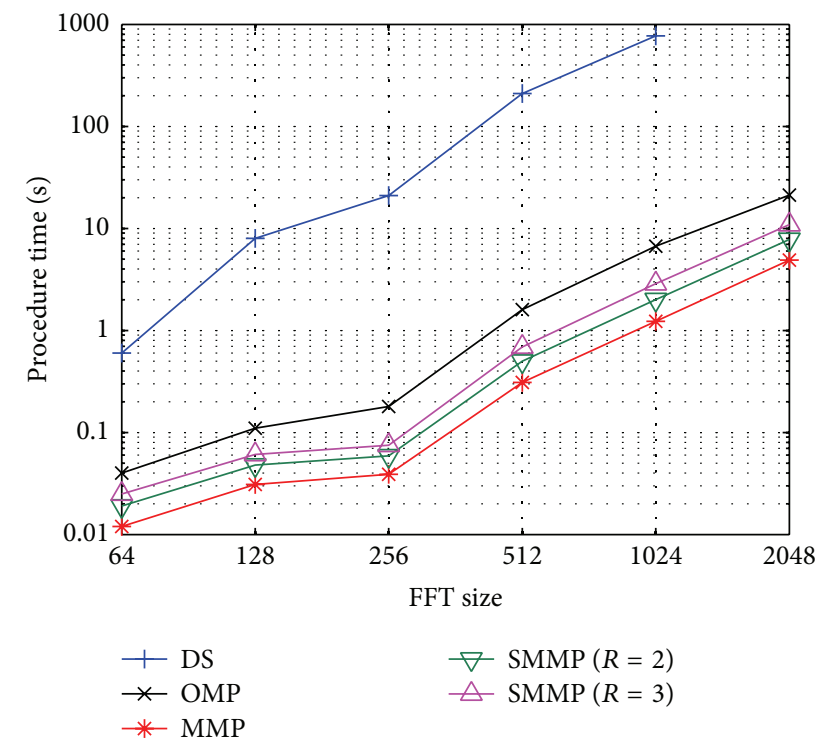

FIGURE 5: Execution time for varying CS methods.

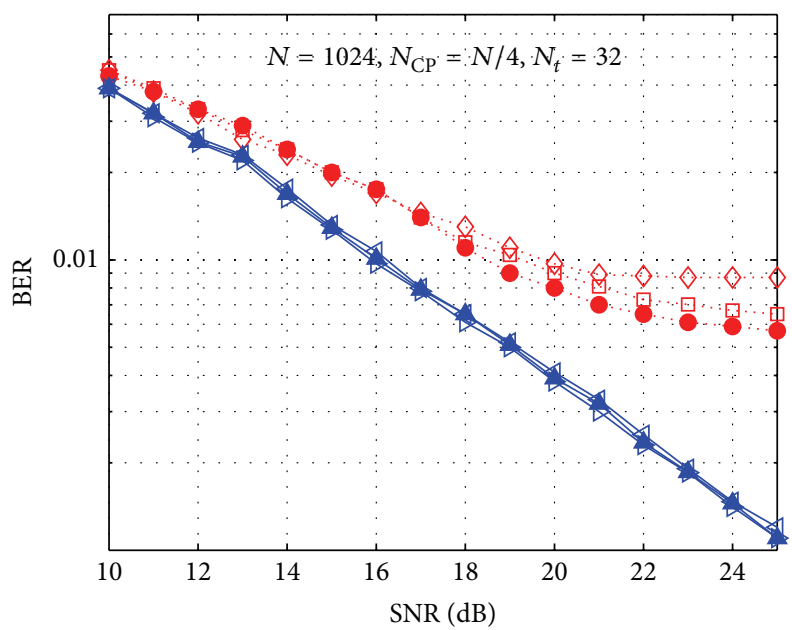

$\diamond . \operatorname{MMP}(\mathrm{Q}=1)\left(F_{d} T_{s}=0.0625\right) \quad \varangle \operatorname{MMP}(\mathrm{Q}=1)(\mathrm{w} / \mathrm{s}$ Doppler $)$

… $\operatorname{MMP}(Q=3)\left(F_{d} T_{s}=0.0625\right) \rightarrow \operatorname{MMP}(\mathrm{Q}=3)(\mathrm{w} / \mathrm{s}$ Doppler $)$

- $\operatorname{OMP}\left(F_{d} T_{s}=0.0625\right) \quad-\mathrm{OMP}(\mathrm{w} / \mathrm{s}$ Doppler $)$

FIGURE 6: Comparision of BER performance between basic MOMP and OMP.

of SMOMP is nearly $\sum_{k=1}^{R} k / R$ times as much as that of the MOMP. In addtion, for giving perceptual comparison of computational complexity, the execution time of OMP and SMOMP has been plotted in Figure 5. With $\rho=0.05$ and $Q=2$, the MOMP steadily costs only about $1 / 3$ execution time as much as the OMP. For SMOMP, the computational complexity increases with the iterative depth $R$.

On the other hand, we also compare the recovery performance between MOMP and OMP in terms of bit error rate (BER) results. Since we do not employ any extra procedure to mitigate the ICI and other interference, the OMP has an upper bound of BER performance. The proposed MOMP can extremely be close to but not attain the bound because of the

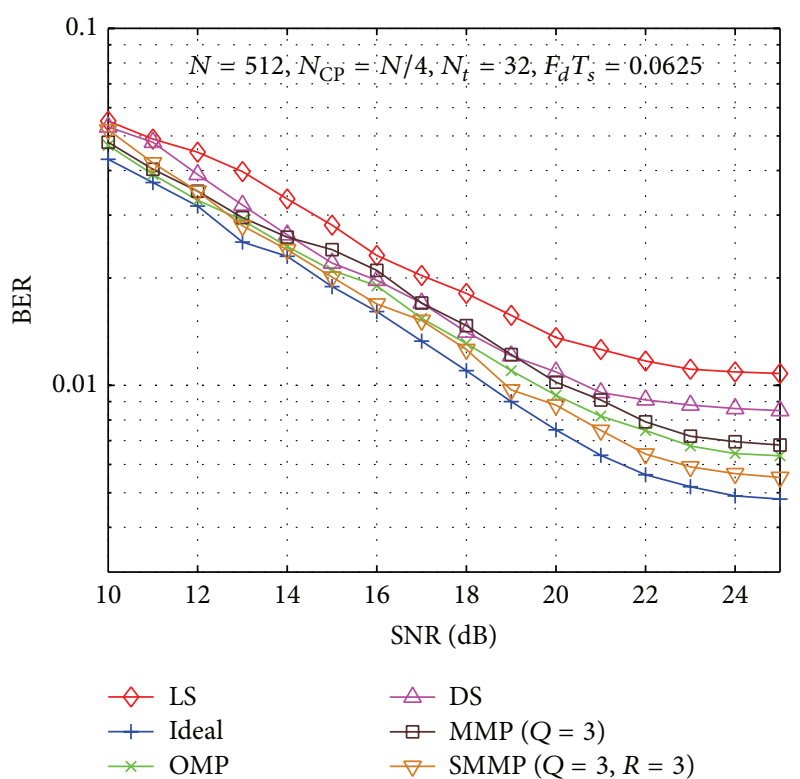

FIGURE 7: BER performance of varying schemes for time-variant doubly selective fading channels without sudden change.

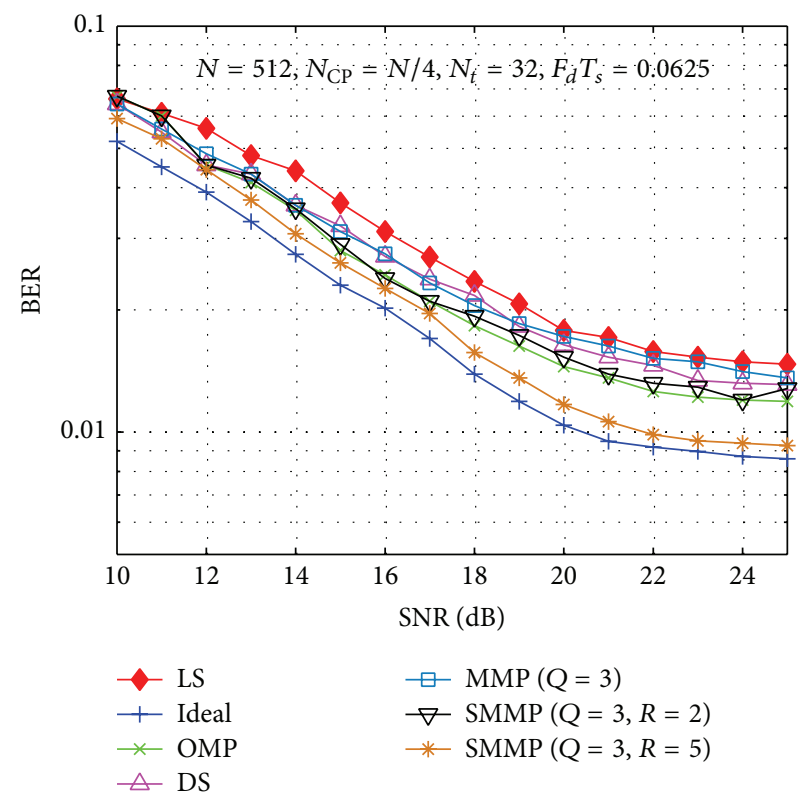

FIGURE 8: BER performance of varying schemes for time-variant fast fading channels with sudden changes.

sparse searching index. For flat fading channels without the effect of Doppler shift, the performance difference between OMP and MOMP is nearly invisible, which is shown in Figure 6. While, for fast fading channels with normalized Doppler shift $f_{d} T_{s}=0.0625$, the MOMP has a slight performance loss comparing with OMP. Also, it is easily noticed that increasing the extending parameter $Q$ can effectively enlarge the searching range of the sensing matrix to improve the MOMP's performance. As $Q=3$ in Figure 6, the BER performance of MOMP is approximate to OMP's 


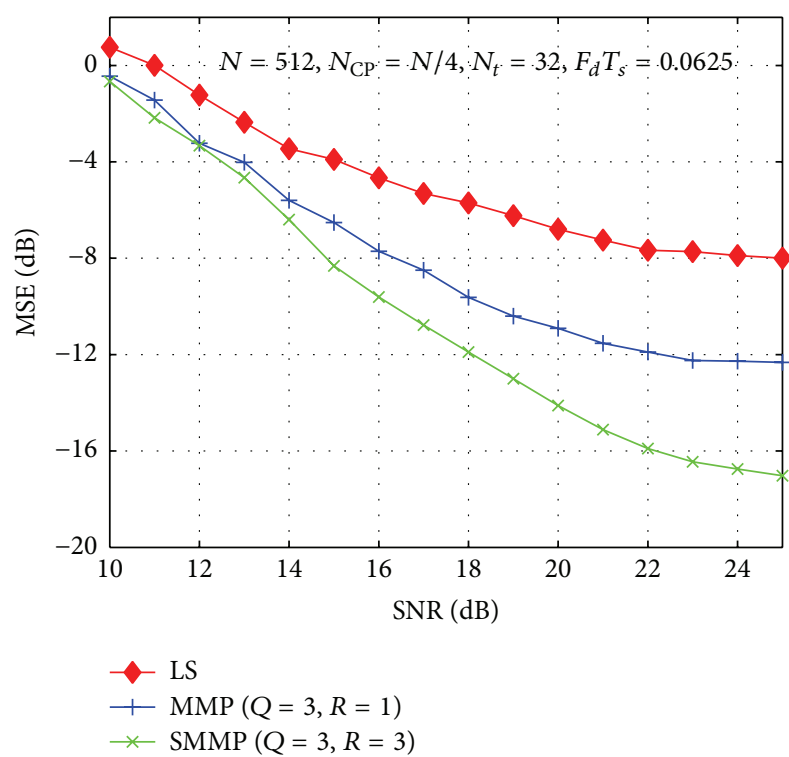

FIGURE 9: MSE performance of the proposed scheme for timevariant fast fading channels without sudden changes.

bound. So setting $Q$ as an appropriate value is an effective way to achieve a trade-off between complexity and accuracy. In addition, Figure 7 shows the BER performance of varying channel estimation schemes for time-variant doubly selective channels without sudden change. All the schemes have a BER performance lower bound, where the conventional LS is the worst one. Using the BER lower bound as a comparison index, the OMP can beat the original MOMP but lose to the SMOMP. The similar comparison between varying schemes is conducted for time-variant fast fading channels with sudden change of $5 \%$ jumping probability of channel coefficients, which is shown in Figure 8 . The relative BER performance significantly degrades for all the schemes. In this case, the advantage of SMOMP is clearer than the original MOMP, as the iterative depth $R=5$. In both Figures 7 and 8 , the performance of DS is really not splendid. Actually, besides DS, some other BP based schemes have also been proposed, which may have better performance for fast fading channels. However, considering the keynote of MP based schemes in this paper, we only select the DS as a typical example of BP based schemes. As a result, the SMOMP shows its robustness regardless of sudden change. To demonstrate the prooposed scheme's performance further, the MSE performance of the estimated matrix channel is also compared between the proposed scheme and the original LS based scheme. It is shown in Figure 9 that the MSE performance has the similar simulation curves with the BER performance. On the other hand, we notice that SMOMP's performance is dominated by the appropriate choice of adaptive parameters that are empirical values now.

\section{Conclusions}

In this paper, we have further improved the original MOMP by using adaptive sliding windows. The proposed slidingMOMP has more robust recovery performance and relatively low computational complexity. The original MOMP requires only $1 / 3$ computational cost to obtain the similar BER performance of the OMP. Adjusting the extending parameter can make the MOMP extremely approximate the OMP's bound. Although the computational complexity increases multiply in terms of the iterative depth, the SMOMP has better BER performance than the conventional OMP. Meanwhile, the increased cost is still under control and lower than the OMP, when the adaptive parameters are set to an appropriate value. We think that the proposed SMOMP is a potential scheme as a low-complexity and high-performance candidate of channel estimators for ISDB-T system.

\section{Competing Interests}

The authors declare that they have no competing interests.

\section{Acknowledgments}

This work has been supportd by the Central State-Owned Captital Management and Budget Project (no. 2013-470), the National Natural Science Foundation of China (no. 61172089), the China Postdoctoral Science Foundation (no. 2014M562100), the Hunan Provincial Science and Technology Project Funds (no. 2014WK3001 and no. 2015JC3053), and the Fundamental Research Funds for the Central Universities (no. 2014-004).

\section{References}

[1] Associtation of Radio Industries and Businesses, Transmission System for Digital Terrestrial Television Broadcasting, ARIB Standard STD-B31 Version. 1.9, 2010.

[2] D. L. Donoho, "Compressed sensing," IEEE Transactions on Information Theory, vol. 52, no. 4, pp. 1289-1306, 2006.

[3] E. J. Candès, J. Romberg, and T. Tao, "Robust uncertainty principles: exact signal reconstruction from highly incomplete frequency information," IEEE Transactions on Information Theory, vol. 52, no. 2, pp. 489-509, 2006.

[4] M. L. Mekhalfi, F. Melgani, Y. Bazi, and N. Alajlan, "A compressive sensing approach to describe indoor scenes for blind people," IEEE Transactions on Circuits and Systems for Video Technology, vol. 25, no. 7, pp. 1246-1257, 2015.

[5] C. Chen, Y. Li, and J. Huang, "Forest sparsity for multi-channel compressive sensing," IEEE Transactions on Signal Processing, vol. 62, no. 11, pp. 2803-2813, 2014.

[6] A. Garcia-Rodriguez and C. Masouros, "Low-complexity compressive sensing detection for spatial modulation in large-scale multiple access channels," IEEE Transactions on Communications, vol. 63, no. 7, pp. 2565-2579, 2015.

[7] C. Zhang, J. Xiao, and Y. Zhang, "Blind compressive sensing detection of watermark coded by limited-random sequence," IEICE Transactions on Fundamentals of Electronics Communications and Computer Sciences A, vol. 98, no. 8, pp. 1747-1750, 2015.

[8] C. R. Berger, Z. Wang, J. Huang, and S. Zhou, "Application of compressive sensing to sparse channel estimation," IEEE Communications Magazine, vol. 48, no. 11, pp. 164-174, 2010.

[9] H. Furudate, M. Umeda, T. Iwamatsu, Z. Ma, T. Sato, and M. Okada, "An application of matching pursuit based channel 
estimation for FODM systems," ITE Technical Report, vol. 37, no. 14, pp. 61-65, 2013.

[10] W. U. Bajwa, J. Haupt, A. M. Sayeed, and R. Nowak, "Compressed channel sensing: a new approach to estimating sparse multipath channels," Proceedings of the IEEE, vol. 98, no. 6, pp. 1058-1076, 2010.

[11] C. R. Berger, S. Zhou, J. C. Presig, and P. Willett, "Sparse channel estimation for multicarrier underwater acoustice communication: from subspace methods to compressed sensing," IEEE Transactions on Signal Processing, vol. 98, no. 6, pp. 1058-1076, 2010.

[12] S. G. Mallat and Z. Zhang, "Matching pursuits with timefrequency dictionaries," IEEE Transactions on Signal Processing, vol. 41, no. 12, pp. 3397-3415, 1993.

[13] J. A. Tropp and A. C. Gilbert, "Signal recovery from random measurements via orthogonal matching pursuit," IEEE Transactions on Information Theory, vol. 53, no. 12, pp. 4655-4666, 2007.

[14] T. Sato, Z. Ma, T. Higashino, and M. Okada, "Low complexity algorithm for leaky coaxial cable based intruder detection system," in Proceedings of the International Conference on Embedded Systems and Intelligent Technology (ICESIT '13), Nong Khai, Thailand, January 2013.

[15] Z. Ma, H. Liu, T. Higashino et al., "Low complexity channel estimation for ISDB-T over doubly-selective fading channels," in Proceedings of the International Symposium on Intelligent Signal Processing and Communication Systems (ISPACS '13), pp. 114-118, New Taibei, Taiwan, November 2013.

[16] J. Meng, W. Yin, Y. Li, N. T. Nguyen, and Z. Han, “Compressive sensing based high-resolution channel estimation for OFDM system," IEEE Journal on Selected Topics in Signal Processing, vol. 6, no. 1, pp. 15-25, 2012.

[17] R. Paderna, T. Higashino, M. Okada, and T. Iwamatsu, "Improved channel estimation for ISDB-T using modified orthogonal matching pursuit over fractional delay channel," in Proceedings of the International Symposium on Communications and Information Technologies (ISCIT '14), pp. 336-340, Incheon, Republic of Korea, September 2014.

[18] R. Paderna, T. Higashino, and M. Okada, "Improved channel estimation for ISDB-T using Modified Orthogonal Matching Pursuit over fractional delay TU6 channel," in Proceedings of the Asia-Pacific Signal and Information Processing Association Annual Summit and Conference (APSIPA '14), pp. 1-5, IEEE, Siem Reap, Cambodia, December 2014.

[19] G. B. Giannakis and C. Tepedelenlioglu, "Basis expansion models and diversity techniques for blind identification and equalization of time-varying channels," Proceedings of the IEEE, vol. 86, no. 10, pp. 1969-1986, 1998.

[20] W. U. Bajaw, J. Haupt, G. Raz, and R. Nowak, "Compressed channel sensing," in Proceeding of the 42nd Annual Conference on Information Sciences and Systems (CISS '08), pp. 1-6, Princeton, NJ, USA, May 2008.

[21] S. Kunis and H. Rauhut, "Random sampling of sparse trigonometric polynomials, II. Orthogonal matching pursuit versus basis pursuit," Foundations of Computational Mathematics, vol. 8, no. 6, pp. 737-763, 2008.

[22] G. E. Pfander, H. Rauhut, and J. Tanner, "Identification of matrices having a sparse representation," IEEE Transactions on Signal Processing, vol. 56, no. 11, pp. 5376-5388, 2008.

[23] E. J. Candès, "The restricted isometry property and its implications for compressed sensing," Comptes Rendus Mathematique, vol. 346, no. 9-10, pp. 589-592, 2008.
[24] P. J. Bickel, Y. Ritov, and A. B. Tsybakov, "Simultaneous analysis of lasso and dantzig selector," The Annals of Statistics, vol. 37, no. 4, pp. 1705-1732, 2009.

[25] Y. Mostofi and D. C. Cox, "ICI mitigation for pilot-aided OFDM mobile systems," IEEE Transactions on Wireless Communications, vol. 4, no. 2, pp. 765-774, 2005.

[26] C. T. Kelley, Iterative Methods for Linear and Nonlinear Equations, Frontiers in Applied Mathematics, Philadelphia, Pa, USA, 1995.

[27] 3rd Generation Partnership Project, “Technical specification group GSM/EDGE radio access network, radio transmission and reception," Tech. Rep. 3GPP TS 05.05 V8.20.0, 2005.

[28] K. G. Murty, Linear Programming, John Wiley \& Sons, New York, NY, USA, 1983.

[29] B. G. Dantzig and M. N. Thapa, Linear Programming 2: Theory and Extensions, Springer, Berlin, Germany, 2003. 


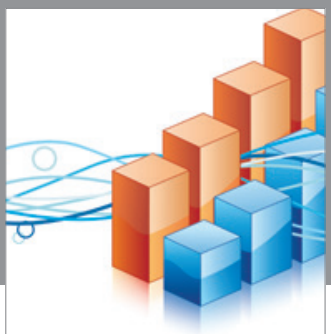

Advances in

Operations Research

vatem alat4

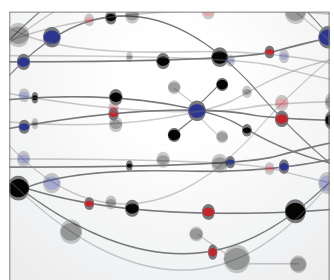

\section{The Scientific} World Journal
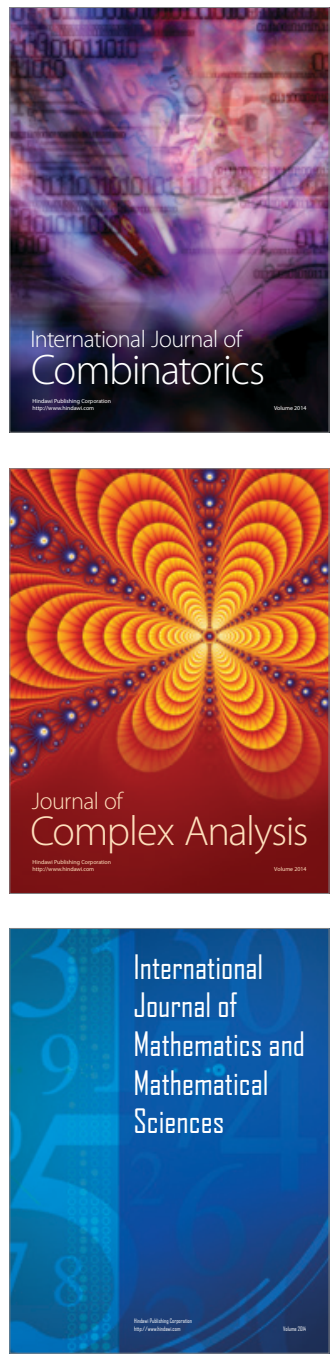
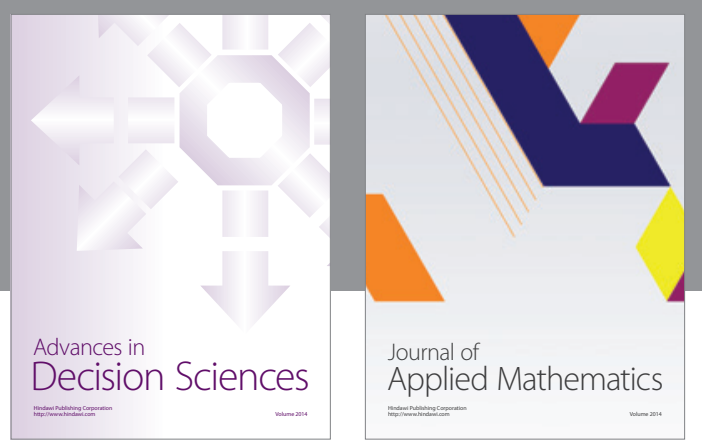

Algebra

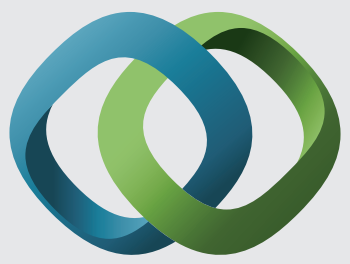

\section{Hindawi}

Submit your manuscripts at

http://www.hindawi.com
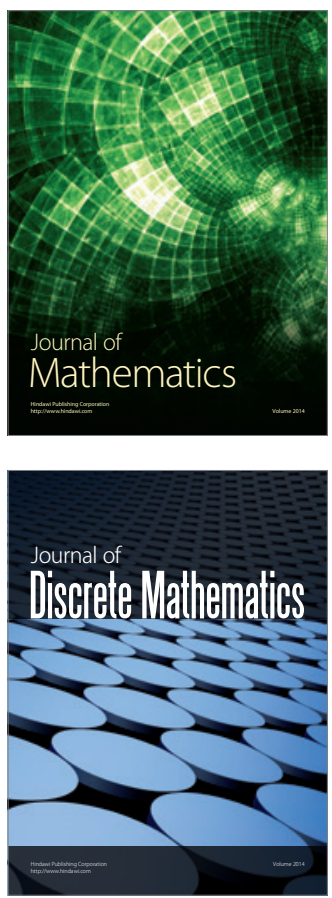

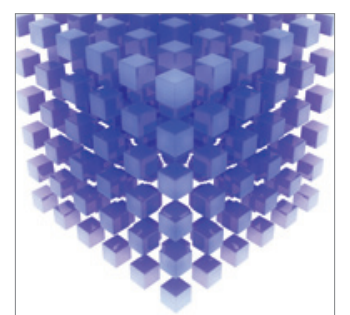

Mathematical Problems in Engineering
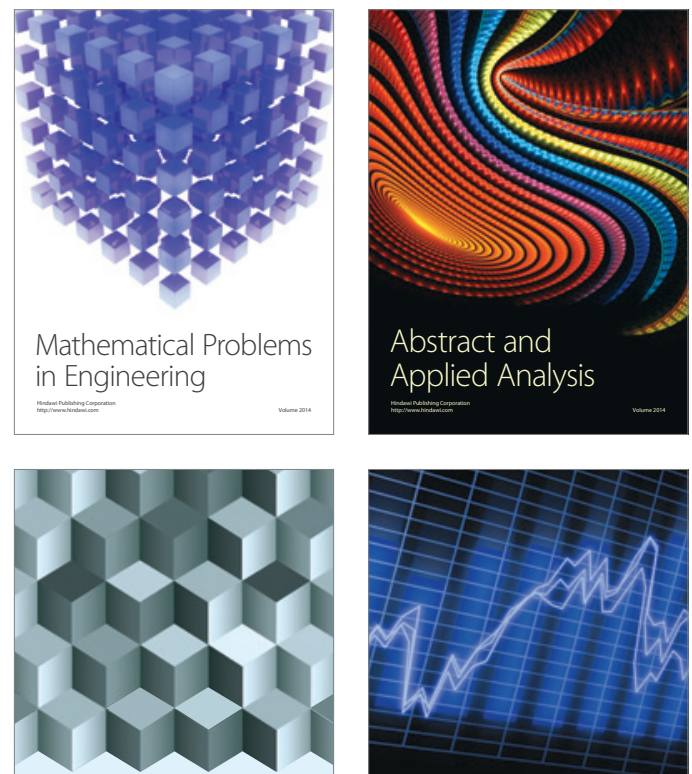

Journal of

Function Spaces

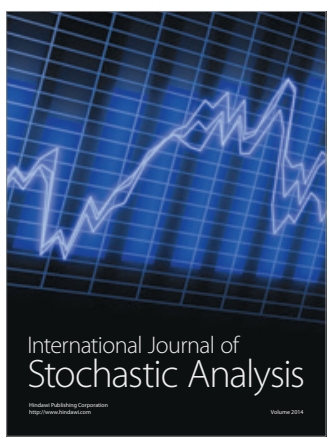

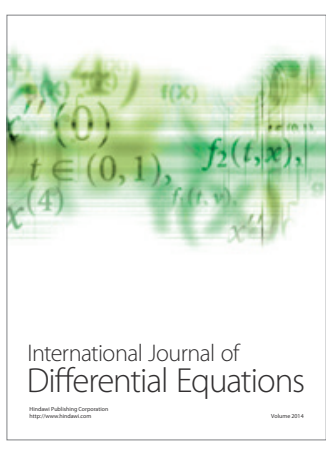
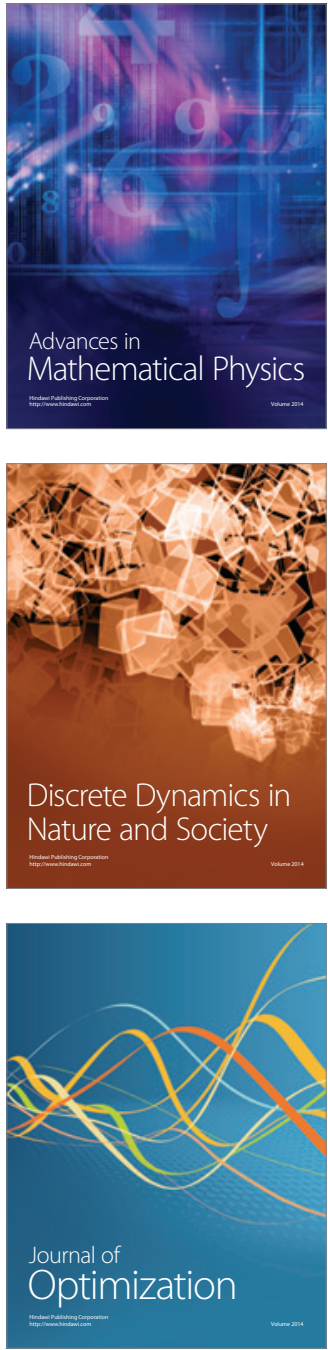